\title{
PREVALÊNCIA DE INCONTINÊNCIA URINÁRIA E ASSOCIAÇÃO COM A OBESIDADE EM MULHERES CADASTRADAS EM UMA UNIDADE DOCENTE ASSISTENCIAL DE MACEIÓ- AL
}

\section{ARTIGO ORIGINAL}

BEZERRA, Ana Clarissa Santos ${ }^{1}$

LIMA, Isabella Karoline Moreira Ribeiro ${ }^{2}$

SOUTINHO, Renata Sampaio Rodrigues ${ }^{3}$

BEZERRA, Ana Clarissa Santos. LIMA, Isabella Karoline Moreira Ribeiro. SOUTINHO, Renata Sampaio Rodrigues. Prevalência de incontinência urinária e associação com a obesidade em mulheres cadastradas em uma unidade docente assistencial de Maceió- AL. Revista Científica Multidisciplinar Núcleo do Conhecimento. Ano 05, Ed. 10, Vol. 09, pp. 91-113. Outubro de 2020. ISSN: 24480959, Link de acesso: https://www.nucleodoconhecimento.com.br/saude/obesidadeem-mulheres

\section{RESUMO}

A incidência de sobrepeso e obesidade assim como marcadores de Doenças Crônicas Não Transmissíveis (DCNT) cresceu nas últimas décadas. A obesidade além de estar associada às doenças crônicas não transmissíveis como diabetes e hipertensão, pode ser consideradas um fator de risco para o desenvolvimento de desordens nas estruturas de sustentação das vísceras abdomino-pélvicas podendo levar a complicações como a incontinência urinária (IU) e os prolapsos genitais. A associação entre incontinência e obesidade ainda é pouco estudada. Devido a relevância do tema,

\footnotetext{
${ }^{1}$ Pós-graduada em Terapia Manual, Graduada em Fisioterapia.

2 Graduada em Fisioterapia.

${ }^{3}$ Orientadora. Mestre em Fisioterapia. Graduada em Fisioterapia.
} 
foi proposto este estudo que teve objetivo investigar a prevalência de incontinência urinária em mulheres cadastradas em uma unidade assistencial da cidade de Maceió e verificar a associação com a obesidade. Trata-se de um estudo observacional, analítico de caráter transversal, com mulheres entre 18 e 60 anos, cadastradas em uma Unidade Docente Assistencial da Cidade de Maceió, no período de julho a setembro de 2017. Foram excluídas as mulheres com gravidez suspeita ou confirmada, ou que possuíssem doença neurológica que apresentassem como sequela dificuldade de compreensão, audição ou fala que impossibilitassem responder ao questionário. Participaram da pesquisa, 109 mulheres, com média de idade de $38,1 \pm 12,3$, variando entre a idade mínima de 18 e a máxima de 59 anos. A prevalência de IU auto referida nesta população foi de $17,43 \%$, não tendo sido encontrada associação com a obesidade. Foi possível concluir que a prevalência da IU está dentro do percentual descrito na literatura e que mesmo não tendo sido encontrada associação estatisticamente significativa entre IU e obesidade, o número de mulheres incontinentes, obesas ou com sobrepeso é alto. Além disso, a IU está cada vez mais presente em mulheres jovens, assim como a obesidade, como pode ser confirmado nesse estudo.

Palavras-Chave: Incontinência urinária, obesidade, mulheres.

\section{INTRODUÇÃO}

A incidência de sobrepeso e obesidade assim como marcadores de Doenças Crônicas Não Transmissíveis (DCNT) cresceu nas últimas décadas. A obesidade não é uma doença singular, é um problema de saúde pública, já que é considerada um fator de risco para outras morbidades como diabetes e doenças cardiovasculares, causas importantes de mortalidade em adultos, que aumentam proporcionalmente ao ganho de peso (SARTORI; DE SOUZA; CARNEIRO, 2011; OLIVEIRA et. al., 2010; VIECELLI et. al., 2009).

A obesidade além de estar associada às doenças crônicas não transmissíveis como diabetes e hipertensão, pode ser considerada um fator de risco para 0 desenvolvimento de desordens nas estruturas de sustentação das vísceras abdomino- 
pélvicas podendo levar a complicações como a incontinência urinária e os prolapsos genitais (DA COSTA; MACHADO; CORDÁS, 2010; FORTI et al., 2011; PIRES, 2013).

A Incontinência Urinária (IU) é definida como uma condição em que ocorre perda involuntária de urina. Esta condição é responsável por transtornos que levam a problemas higiênicos, disfunções sexuais, baixo desempenho profissional, impactando de forma negativa na qualidade de vida. Segundo a Sociedade Internacional de Continência, a IU pode ser classificada em Incontinência Urinária de Esforço (IUE), Incontinência Urinária de Urgência (IUU) e Incontinência Urinaria Mista (IUM) (DE CARVALHO et. al., 2013).

A incontinência urinária de esforço (IUE) é considerado o tipo de incontinência mais comum em mulheres jovens, afetando $18 \%$ das mulheres com idade acima dos 30 anos. Em mulheres obesas, é o tipo mais prevalente, sendo caracterizada pela perda involuntária de urina, quando a pressão intra-vesical excede a pressão de fechamento uretral máxima na ausência de contração do músculo detrusor. Nesse tipo de incontinência é muito comum ocorrer perda de urina em situações como espirro, tosse, risada ou ainda durante a prática de atividades físicas (FROHME et. al., 2014).

A associação entre incontinência e obesidade ainda é pouco estudada. Alguns autores constataram que a obesidade é considerada um fator de risco relevante para o desenvolvimento de IUE, principalmente em mulheres na faixa etária entre 37 a 54 anos. WING e colaboradores (2010) relatam que a perda de peso em mulheres com sobrepeso ou obesas, exerce efeito positivo na redução da frequência dos episódios de IUE.

O mecanismo que explica a relação da IU com a obesidade ainda não é bem estabelecido. Especula-se que o excesso de peso aumente a pressão intraabdominal, que é transferida à bexiga, aumentando a pressão intravesical e a mobilidade uretral, levando à IU. Além disso, a obesidade pode afetar a função neuromuscular do trato geniturinário, contribuindo para as queixas urinárias (VIECELLI et. al., 2009). 
Considerando que o número de mulheres obesas com IU ainda é um dado subestimado, e que conhecer se existe associação ou não destas duas condições é útil para elaborar estratégias de tratamento específico para esta população, foi proposto este estudo que teve objetivo investigar a prevalência de incontinência urinária em mulheres cadastradas em uma unidade assistencial da cidade de Maceió e verificar a associação com a obesidade.

\section{MATERIAL E MÉTODO}

Trata-se de um estudo observacional, analítico de caráter transversal, com mulheres ente 18 e 60 anos, cadastradas em uma Unidade Docente Assistencial da Cidade de Maceió, no período de julho a setembro de 2017. Foram excluídas mulheres gestantes ou com suspeita de gravidez, ou que possuíssem doença neurológica que apresentasse como sequela dificuldade de compreensão, audição ou fala que impossibilitassem responder ao questionário.

O cálculo do tamanho da amostra foi feito consultando o site do LEE - Laboratório de Epidemiologia e Estatística, considerando prevalência de incontinência urinária de 17 \%, precisão absoluta de 5 e nível de significância de 5\%. O tamanho da amostra calculada foi de 217 , acrescentando-se $20 \%$ para perdas e recusas; o tamanho amostral mínimo foi de 261 mulheres.

Para obtenção da amostra, foi considerada a divisão da Unidade Docente Assistencial em 6 microáreas. A coleta foi iniciada na $1^{1}{ }^{a}$ microárea e somente após a finalização dos domicílios desta região é que era dado sequência as próximas microáreas.

Dentro de cada microárea, foi feita a identificação dos setores censitários, sendo excluídos os setores comerciais.

O processo de seleção dos domicílios foi iniciado por conglomerados. Foi percorrido um determinado número de domicílios, realizando-se sistematicamente as entrevistas das mulheres, pela própria pesquisadora e por voluntárias graduandas da área de saúde, previamente treinadas. 
Caso não fossem encontradas mulheres com idade entre 18 e 60 anos nos domicílios alvos de pesquisa, a pesquisadora dava continuidade ao domicílio mais próximo, de forma contínua, até que fosse identificada uma mulher elegível. Se caso fosse encontrado um domicílio que possuísse uma mulher elegível, e a mesma não estivesse presente no momento, era marcado um outro tempo de visita, no máximo duas vezes, para considerá-lo como perda. Frente à ocorrência de mais de uma mulher com idade entre 18 e 60 anos residindo no domicílio, todas eram entrevistadas.

Após a assinatura do Termo de Consentimento Livre e Esclarecido - TCLE (apêndice A), baseado na resolução 466/12, do Conselho Nacional de Saúde, do Ministério da Saúde (CNS/MS), através de uma entrevista estruturada, de modo individualizado, os dados foram colhidos nos domicílios de cada participante por apenas um pesquisador.

As variáveis independentes, tais como aspectos sociodemográficos e econômicos, doenças autorreferidas, hábitos de vida e antecedentes gineco-obstétricos, foram coletadas por meio de um formulário de coleta de dados.

As variáveis independentes do estudo incluíram: idade, escolaridade, estado civil, ocupação, renda per capita, paridade, tipo de parto, realização de cirurgia uroginecológica prévia, presença de doença crônica e tipo de doença crônica, prática de atividade física regular e uso de medicação diária.

A variável dependente "incontinência urinária" foi obtida através da pergunta:

" a senhora teve perda urinária em qualquer ocasião nos últimos doze meses?", sendo possível obter dados sobre a incontinência autorreferida.

Também foi realizada a medida da circunferência abdominal através de uma fita métrica, sendo solicitado a voluntária a ficar de pé e a fita métrica era colocada na linha umbilical, sendo a medida obtida em centímetros. Esta medida mostra o conteúdo de gordura visceral e de acordo com as Diretrizes Brasileiras de Obesidade (2010), medida igual ou maior que $80 \mathrm{~cm}$, em mulheres, representa risco aumentado e maior ou igual a 88 representa risco aumentado de forma substancial para o desenvolvimento de doença cardiovascular. Baseado no estudo feito por Oliveira 
(2010), o presente estudo considerou como obesidade abdominal uma circunferência acima de $88 \mathrm{~cm}$.

Para verificação da altura, foi utilizado um estadiômetro usado em base sem desníveis numa superfície plana solicitando a voluntária que ficasse na posição de ortostase.

O índice de massa corporal (IMC) foi calculado a partir das medidas de peso e altura, através da seguinte fórmula IMC = peso $(\mathrm{kg}) /$ altura $^{2}(\mathrm{~cm})$. E foram adotados os seguintes pontos de corte de IMC, baixo peso (IMC < 18,5); eutrofia (IMC 18,5-24,99); sobrepeso (IMC 25-29,99) e obesidade (IMC $\geq 30,00)$.

Foi realizado um registro dos resultados no banco de dados e suas respectivas análises. As variáveis quantitativas foram exibidas em média e desvio padrão, enquanto as quantitativas foram realizadas em forma de tabela de frequência.

Análises bivariadas foram realizadas para identificar uma possível associação entre a IU e a obesidade pelo IMC e pela medida da circunferência abdominal.

\section{RESULTADOS}

Os resultados aqui apresentados se referem a análise parcial dos dados, pois não foi possível concluir a coleta em tempo devido a problemas na microárea onde estava sendo realizada a pesquisa, totalizando $41,7 \%$ do cálculo amostral.

Participaram da pesquisa, 109 mulheres, com média de idade de 38,1 $\pm 12,3$, variando entre a idade mínima de 18 e a máxima de 59 anos.

A prevalência de IU autorreferida nesta população foi de $17,43 \%(n=19)$, sendo que $21 \%(n=4)$ referiram ter perda urinária aos esforços, $26,3 \%(n=5)$ relataram ter perda associada a urgência miccional e a maioria $52,7 \%(n=10)$ sinalizou que a perda acontece tanto aos esforços quanto com a urgência, sendo sugestivo de incontinência urinária mista. 
Quanto ao tempo de incontinência, $52,7 \%(n=10)$ referiram que as queixas iniciaram há menos de 1 ano e 47,3\% (n=9) há mais de 1 ano. Destas, 47,3\% (n=9) relataram ter que usar protetor higiênico diário enquanto $52,7 \%(n=10)$ não fazem uso do protetor. Apenas $1(5,26 \%)$ participante possuía diagnóstico baseado no estudo urodinâmico e 2 (10,52\%) delas afirmaram já ter realizado algum tratamento para esta condição.

A tabela 1 demonstra o perfil das mulheres participantes da pesquisa, quanto aos dados sociodemográficos, econômicos e hábitos de vida, de acordo com a presença ou não da incontinência urinária.

Tabela 1 - Perfil das participantes da pesquisa, quanto aos dados sóciodemográficos, econômicos e hábitos de vida, de acordo com a presença ou não da incontinência urinária.

\begin{tabular}{|c|c|c|c|c|c|c|c|c|}
\hline & & $\begin{array}{l}\text { Sem } \\
\text { IU }\end{array}$ & & $\begin{array}{l}\text { Com } \\
\text { IU }\end{array}$ & & TOTAL & & $\mathbf{p}^{\star \star}$ \\
\hline \multicolumn{2}{|l|}{ Características } & $n=90$ & $\%$ & $\mathrm{n}=19$ & $\%$ & $n=109$ & $\%$ & \\
\hline \multicolumn{9}{|l|}{ Estado civil } \\
\hline & Solteiras & 33 & 30,3 & 7 & 6,4 & 40 & 36,7 & 0,14 \\
\hline & Casadas & 50 & 45,8 & 11 & 10 & 61 & 55,9 & \\
\hline & Divorciadas & 3 & 2,8 & 0 & 0 & 3 & 2,8 & \\
\hline & Viúvas & 3 & 2,8 & 1 & 0,9 & 4 & 3,6 & \\
\hline \multicolumn{2}{|l|}{ Ocupação } & & & & & & & 0,27 \\
\hline & Do lar & 46 & 42,2 & 9 & 8,3 & 55 & 50,5 & \\
\hline & Empregada & 20 & 18,3 & 6 & 5,6 & 26 & 23,9 & \\
\hline & Estudante & 7 & 6,4 & 1 & 0,9 & 8 & 7,3 & \\
\hline & Desempregada & 17 & 15,6 & 3 & 2,7 & 20 & 18,3 & \\
\hline \multicolumn{2}{|c|}{ Grau de escolaridade } & & & & & & & 0,33 \\
\hline & $\begin{array}{l}\text { Não } \\
\text { alfabetizadas }\end{array}$ & 9 & 8,3 & 1 & 0,9 & 10 & 9,2 & \\
\hline
\end{tabular}


https://www.nucleodoconhecimento.com.br

\begin{tabular}{|c|c|c|c|c|c|c|c|c|}
\hline & $\begin{array}{l}\text { Ensino } \\
\text { fundamental }\end{array}$ & 40 & 36,7 & 11 & 10 & 51 & 46,7 & \\
\hline & Ensino médio & 31 & 28,5 & 6 & 5,6 & 37 & 34,1 & \\
\hline & Ensino superior & 10 & 9,1 & 1 & 0,9 & 11 & 10 & \\
\hline \multicolumn{2}{|l|}{ Renda per capita } & & & & & & & 0,29 \\
\hline & Até 1 salário & 67 & 61,5 & 13 & 11,9 & 80 & 73,4 & \\
\hline & De 1 a 3 & 17 & 15,5 & 5 & 4,6 & 22 & 20,1 & \\
\hline & Acima de 3 a 5 & 6 & 5,6 & 1 & 0,9 & 7 & 6,5 & \\
\hline & Acima de 5 & 0 & 0 & 0 & 0 & 0 & 0 & \\
\hline \multicolumn{2}{|c|}{ Doenças referidas* } & & & & & & & 0,33 \\
\hline & Hipertensão & 23 & 21,1 & 6 & 5,5 & 29 & 26,6 & \\
\hline & Diabetes & 4 & 3,6 & 2 & 1,8 & 6 & 5,5 & \\
\hline & Outras & 0 & 0 & 1 & 0,9 & 1 & 0,9 & \\
\hline & Nenhuma & 63 & 57,8 & 10 & 9,2 & 73 & 67 & \\
\hline \multirow[t]{3}{*}{$\begin{array}{l}\text { Medicação de } \\
\text { rotina }\end{array}$} & & & & & & & & 0,34 \\
\hline & Sim & 34 & 31,2 & 10 & 9,2 & 44 & 40,4 & \\
\hline & Não & 56 & 51,3 & 9 & 8,3 & 65 & 59,6 & \\
\hline \multirow[t]{3}{*}{$\begin{array}{l}\text { Atividade } \\
\text { Física }\end{array}$} & & & & & & & & 0,25 \\
\hline & Sim & 18 & 16,5 & 5 & 4,6 & 23 & 21,2 & \\
\hline & Não & 72 & 66 & 14 & 12,8 & 86 & 78,8 & \\
\hline
\end{tabular}

Fonte: Dados da pesquisa, 2017. $n=$ número de participantes. \%=percentual. *permite mais de uma alternativa. $p^{\star \star}$ menor ou igual a 0,05

Quanto ao estado civil verificou-se que a maioria das participantes incontinentes eram casadas $(11 / 10 \%)$ e em seguida as solteiras $(7 / 6,4 \%)$ sendo em menor número as viúvas (1/0,9\%). Entre as sem queixas de perda urinária, houve também predomínio de casadas $(50 / 45,8 \%)$ seguido de solteiras $(33 / 30,3 \%)$. 
Sobre a ocupação, a maior parte das com incontinência e sem incontinência eram do lar, seguido de empregadas (20/18,3\% - 6/5,6\%), desempregadas (17/15,6\% - 3/2,7\%) e estudantes $(7 / 6,4 \%-1 / 0,9 \%)$ para as sem incontinência e com incontinência respectivamente.

No que se refere ao grau de escolaridade verificou-se que o maior número entre as incontinentes (11/10\%) e continentes (40/36,7\%) eram de mulheres que estudaram até $\mathrm{o}$ ensino fundamental, seguido das que referiram estudar até o ensino médio $(6 / 5,6 \%-31 / 28,5 \%)$.

No que diz respeito a renda per capita tanto entre as incontinentes (13/11,9\%) e não incontinentes $(67 / 61,5 \%)$ houve predominância de até 1 salário mínimo, seguido de 1 a 3 salários (5/4,6\% - 17/15,5\%) e acima de 3 a 5 salários (1/0,9\% - 6/5,6\%).

Sobre as doenças referidas, a maioria das entrevistadas referiu não ter nenhuma doença em ambos os grupos. Entre as que refeririam ter doenças, a hipertensão arterial seguidas de diabetes, foram as mais citadas entre as incontinentes e continentes. No grupo de incontinentes a maioria faz uso de medicação de rotina $(10 / 9,2 \%)$ e nas sem incontinência 56 (51,3\%) \% não fazem uso de remédios diários.

Quanto a atividade física, verificou-se que em ambos os grupos - com incontinência (14/12,8\%) e sem incontinência (72/66\%), a maioria relatou não ter hábito de realizar exercícios físicos pelo menos três vezes por semana.

$\mathrm{Na}$ tabela 2 são apresentados os dados gineco-obstétricos das participantes do estudo.

Tabela 2: Dados gineco-obstétricos das participantes de acordo com a presença ou não de incontinência urinária.

\begin{tabular}{|c|c|c|c|c|c|c|c|}
\hline \multirow[b]{2}{*}{ Características } & \multicolumn{2}{|c|}{$\begin{array}{l}\text { Com } \\
\text { IU(n=19) }\end{array}$} & \multicolumn{2}{|c|}{$\begin{array}{l}\text { Sem } \\
I U(n=90)\end{array}$} & \multicolumn{2}{|c|}{$\begin{array}{l}\text { Total } \\
(n=109)\end{array}$} & $\mathbf{p}^{\star \star}$ \\
\hline & $\mathrm{N}$ & $\%$ & $\mathrm{~N}$ & $\%$ & $\mathrm{~N}$ & $\%$ & \\
\hline
\end{tabular}




\begin{tabular}{|c|l|l|l|l|l|l|l|l|}
\hline Paridade & & & & & & 0,02 \\
\hline Nulípara & 2 & 1,9 & 18 & 16,6 & 20 & 18,5 & \\
\hline Primípara & 5 & 4,7 & 20 & 18,5 & 25 & 23,2 & \\
\hline Multípara & 12 & 11,1 & 51 & 46,9 & 63 & 58 & \\
\hline \begin{tabular}{|c|c|c|c|c|c|c|c|c|c|c|} 
Tipo de parto \\
Vaginal
\end{tabular} & 10 & & & & & & 0,44 \\
\hline Nenhum & 2 & 1,8 & 18 & 16,5 & 20 & 18,3 & \\
\hline Cesáreo & 3 & 2,7 & 26 & 23,8 & 29 & 26,6 & \\
\hline Ambos & 4 & 3,6 & 15 & 13,7 & 19 & 17,4 & \\
\hline Cirurgias Prévias* & & & & & & & 0,36 \\
\hline Abdominal e pélvica & 1 & 0,9 & 3 & 2,8 & 4 & 3,7 & \\
\hline Pélvica & 6 & 5,5 & 9 & 8,3 & 15 & 13,8 & \\
\hline Abdominal & 2 & 1,8 & 7 & 6,4 & 9 & 8,2 & \\
\hline Períneo & 0 & 0 & 1 & 0,9 & 1 & 0,9 & \\
\hline Nenhuma & 10 & 9,2 & 70 & 64,2 & 80 & 73,3 & \\
\hline
\end{tabular}

Fonte: Dados da pesquisa, 2017. N= número de participantes. \%=percentual.

*permite mais de uma alternativa. $p^{* *}$ menor ou igual a 0,05

Os dados ginecológicos e obstétricos revelaram que quanto à paridade, nas incontinentes houve predominância de multíparas (12/11,1\%) seguido das primíparas (5/4,7\%). Nas continentes também houve a predominância das multíparas (51/46,9\%) seguido das primíparas (20/18,5\%) e nulíparas (18/16,6\%).

Das 17 mulheres incontinentes que pariram, 10(9,3\%) foram por via vaginal, $3(2,7 \%)$ cesáreas e $4(3,6 \%)$ tiveram ambos os partos. Referente as 71 continentes que pariram, $31(28,4 \%)$ foram por via vaginal, 18 (16,5\%) cesáreos e $15(13,7 \%)$ tiveram ambos os tipos de parto.

Quanto à realização de cirurgias uroginecológicas, a maior parte das incontinentes $(10 / 9,2 \%)$ e das continentes $(70 / 64,2 \%)$ relataram não ter realizado nenhuma cirurgia. 
Entre as que realizaram, houve maior predominância em cirurgias pélvicas para ambos os grupos - incontinentes (6/5,5\%) e continentes $(9 / 8,2 \%)$.

Sobre os dados antropométricos, a média da circunferência abdominal encontrada foi de 92,06 $\pm 15,08 \mathrm{~cm}$, sendo que $5(4,6 \%)$ das incontinentes possuíam circunferência abdominal menor ou igual a $88 \mathrm{~cm}$ e $14(12,8 \%)$ maior que $88 \mathrm{~cm}$. Dentre as continentes, $39(35,8 \%)$ possuíam circunferência abdominal menor ou igual a $88 \mathrm{~cm}$ e $51(46,8 \%)$ maior que $88 \mathrm{~cm}$. O peso médio foi de $68,82 \pm 15,94 \mathrm{~kg}$ e a altura média foi de $1,55 \pm 0,06 \mathrm{~m}$.

Quanto ao IMC, destaca-se entre as incontinentes as obesas (12/11\%) seguido das eutróficas (6/5,5\%), sobrepeso (1/0,9\%) e nenhuma com baixo peso. Dentre as continentes, a maioria era eutrófica $(31 / 28,5 \%)$, seguido das com sobrepeso $(29 / 26,7 \%)$, obesas e de baixo peso $(2 / 1,9 \%)$.

A Tabela 3 descreve as características antropométricas da população estudada, de acordo com a presença ou não da incontinência urinária.

Tabela 3 - Características antropométricas da população estudada, de acordo com a presença ou não da incontinência urinária.

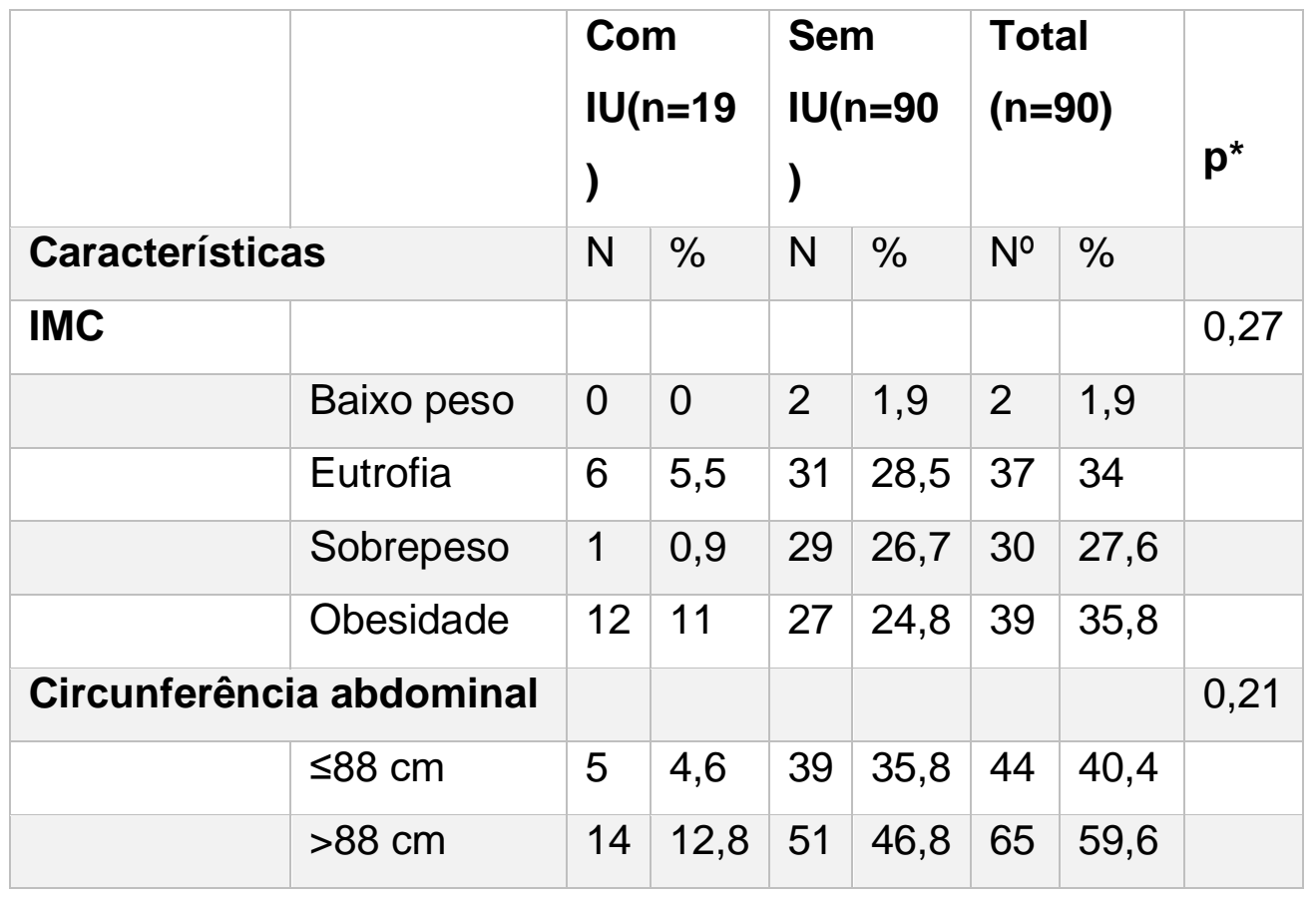


Fonte: Dados da pesquisa, 2017. N= número de participantes. \%=percentual.

$p^{*}$ menor ou igual a 0,05

A análise estatística mostrou correlação estatisticamente significativa entre as variáveis incontinência urinária e paridade.

Em análises bivariadas, considerando-se a associação da IU com o IMC e/ou com a circunferência abdominal constatou-se que não houve associação entre as variáveis estudadas.

\section{DISCUSSÃO}

Os dados sobre prevalência da IU encontrados na literatura são bastante variáveis e revelam um amplo espectro de taxas de prevalência, que de acordo com Dedicação e colaboradores (2008) se deve ao fato de existirem variações nas definições da IU e nas características dos estudos e população alvo.

É fato que, no Brasil, são poucos os estudos sobre a prevalência de IU e os seus fatores de risco. A ampla gama dos estudos realizados neste tema não tem como foco a relação entre o aumento de gordura corporal e a incidência de perdas urinárias, além da possibilidade da ocorrência de uma evolução a incontinência urinária.

Os achados da presente pesquisa foram semelhantes ao estudo de Oliveira (2010), que encontrou uma prevalência de IU também de $17 \%$, sendo a que a maioria apresentava sintomas sugestivos de IUU, enquanto neste trabalho houve maior predominância de IUM. Ambos os estudos diferem do que se supõe a literatura que destaca que o tipo de IU mais frequente em mulheres obesas seria a IUE, sendo justificado por Silva e colaboradores (2011) pois em mulheres obesas há um excessivo acúmulo de gordura corporal que aumenta a pressão intra-abdominal sucedendo uma sobrecarga à musculatura do assoalho pélvico, contribuindo para um suporte anatômico inadequado, o que levaria a uma maior predisposição a perda urinária. 
É importante enfatizar que foi avaliada a IU referida pela participante e apenas 1 delas apresentava exame diagnóstico para esta condição. O diagnóstico de IU envolve a história clínica, o exame físico e o estudo urodinâmico, que de acordo com Feldner Jr (2006), objetiva identificar as causas específicas dos sintomas das pacientes, sendo considerado o melhor método diagnóstico para avaliação da função do trato urinário; porém alguns autores acreditam que o mesmo não é rotineiramente necessário e preferem conduzir suas pacientes baseando-se em sintomas clínicos.

Outro dado a ser destacado é que apenas 2 dentre as incontinentes relataram ter realizado algum tipo de tratamento para esta condição. Um estudo conduzido por $\mathrm{Da}$ Silva e Lopes (2009) apontou que a maior parte das mulheres incontinentes não procuram tratamento pelo fato de achar normal a perda de urina, não a considerar algo importante e a questão de o médico dizer que não seria necessário. Os autores concluíram que o desconhecimento sobre os tipos de tratamento podem contribuir para não procurarem ajuda profissional.

A maioria das entrevistadas não referiu nenhuma doença, porém dentre as incontinentes, muitas relataram ter hipertensão arterial sistêmica seguida de diabetes, comum em mulheres com sobrepeso e obesas e que tem como característica não praticar atividade física regular como a população estudada. Apesar deste não ser um dado estatisticamente significativo para este estudo, sabe-se que alguns medicamentos utilizados rotineiramente por pacientes hipertensos, aumentam a frequência e a urgência urinária, atuando no trato urinário inferior, com a possibilidade de alterar a função vesical piorando ou contribuindo para o aumento da frequência da perda urinária (HIGA; LOPES e REIS, 2008). Tamanini (2009) destaca ainda que uma das doenças mais associadas com a IU é a diabetes.

A variável paridade foi a única que mostrou ter associação estatisticamente significativa com a IU. Segundo Higa; Lopes e Reis (2006), a paridade é reconhecida como o agente que tem maior preocupação em associar-se com o desenvolvimento de IU. Frequentemente a incontinência se faz presente durante a gravidez e quando prevalente há um aumento da paridade, favorecendo a IU. No presente estudo, houve 
maior predomínio de multíparas entre as incontinentes e que tiveram parto por via vaginal.

De acordo com Barbosa e colaboradores (2005) o parto vaginal em primíparas diminui a força muscular do assoalho pélvico e aumenta o risco relativo de força muscular diminuída do assoalho pélvico, após 4 a 6 meses do parto.

Sobre os dados antropométricos, ressalta-se que a população estudada, independente da IU, apresenta medidas elevadas de circunferência abdominal e IMC o que é um dado preocupante já que o sobrepeso e a obesidade aumentaram bastante nos últimos tempos em países desenvolvidos e também nos em desenvolvimento, tornando-se um sério problema de saúde pública pelo fato de existirem diversas consequências da obesidade.

De acordo com Castro e colaboradores (2012), as mulheres obesas têm uma chance maior de desencadear IU principalmente relacionada ao esforço. Para Fitz e colaboradores (2012) a integração existente entre a obesidade e as disfunções do assoalho pélvico, está relacionada com o índice de massa corporal e a pressão intraabdominal, pelo fato da obesidade e sobrepeso atingirem o assoalho pélvico devido ao aumento crônico da pressão intra-abdominal. A obesidade gera acúmulo de gordura no interior do abdome e induz o aumento da pressão intra-abdominal, a qual é transmitida à bexiga, e intra-vesical que fadiga e enfraquece a musculatura do assoalho pélvico, facilitando assim a perda de urina em situações como, tossir, espirrar ou correr.

É válido considerar que mesmo não tendo sido encontrada associação estatisticamente significativa entre a IU e a obesidade, identificamos nesta pesquisa um número considerável de mulheres obesas ou com sobrepeso e com circunferência abdominal acima de $88 \mathrm{~cm}$, sendo necessária uma intervenção para modificação destes indicadores de saúde. 


\section{CONCLUSÃO}

A análise dos resultados parciais desta pesquisa conclui que a prevalência da IU está dentro do percentual descrito na literatura e que mesmo não tendo sido encontrada associação estatisticamente significativa entre IU e obesidade, o número de mulheres incontinentes, obesas ou com sobrepeso é alto.

Além disso, a IU está cada vez mais presente em mulheres jovens, assim como a obesidade, como pôde ser confirmado nesse estudo. Dessa forma, é importante a profilaxia da perda urinária a fim de evitar o aparecimento da mesma e de suas consequências que interferem negativamente na qualidade de vida.

Outros estudos podem ser realizados para acompanhamento das mulheres incontinentes com sobrepeso ou obesas, para avaliar se a perda de peso tem um impacto significativo na redução das queixas urinárias.

\section{REFERÊNCIAS}

BARBOSA, A. et. al. Efeito da via de parto sobre a força muscular do assoalho pélvico. Revista Brasileira de Ginecologia e Obstetrícia, Rio de Janeiro, v.27, n.11,2 005

CASTRO, L. A. et. al. Effects of bariatric surgery on pelvic floor function. Revista ABCD: arquivos brasileiros de cirurgia digestiva, Curitiba, v. 25, n. 4, p. 1-6, 2012

DA COSTA, R., MACHADO, S., CORDÁS, T. Imagem corporal e comportamento sexual de mulheres obesas com e sem transtorno da compulsão alimentar periódica. Revista de Psiquiatria Clínica, v.37, n. 1, São Paulo, 2010.

DA SILVA, L., LOPES, M. H. Incontinência urinária em mulheres: razões da não procura por tratamento. Revista da Escola de Enfermagem da USP, São Paulo, v.43, n.1, 2009 
DE CARVALHO, M., et. al. O impacto da incontinência urinária e seus fatores associados em idosas. Revista Brasileira de Geriatria e Gerontologia, Rio de Janeiro, v.17, n.4, p. 721-730, 2014

DEDICAÇÃO, A. C., et. al. Comparação da qualidade de vida nos diferentes tipos de incontinência urinária feminina. Revista Brasileira de Fisioterapia. São Carlos, n.2, p. 116-122, 2008.

DIRETRIZES BRASILEIRAS DE OBESIDADE. Associação Brasileira para o Estudo da Obesidade e da Síndrome Metabólica. - 3.ed. - Itapevi, São Paulo, 2009.

FELDNER, P., et. al. Diagnóstico Clínico e subsidiário da incontinência urinária. Revista brasileira de Ginecologia e obstetrícia. São Paulo, n. 1, p.54-62, 2006.

FITZ, F. F., et. al. Qual o índice de massa corporal de mulheres com disfunções dos músculos do assoalho pélvico que procuram tratamento fisioterapêutico? Fisioterapia e Pesquisa, São Paulo, v. 19, n. 4, p. 309-313, 2012

FORTI, E. M., et. al. Estudo da mobilidade torácica de pacientes portadoras de obesidade Mórbida, submetidas a gastroplastia com acompanhamento Fisioterapêutico. Revista Eletrônica Inspirar, Curitiba, v. 3 n. 10, 2011.

FROHME, C., et. al. TOT Approach in stress urinary incontinence (SUI) - outcome in obese female. BMC Urology, v.14, n.20, 2014

HIGA, R., LOPES, M. H., REIS, M. J. Fatores de risco para incontinência urinária na mulher. Revista da Escola de Enfermagem da USP. São Paulo, n. 1, p. 187-192, 2008

OLIVEIRA E. et. al. Influência do índice de massa corporal na incontinência urinária feminina. Rev. Bras. Ginecol. Obstet., Rio de Janeiro, v. 32, n. 9, p. 454-458, 2010.

OLIVEIRA, J. Prevalência da incontinência urinária e sua associação com a obesidade em mulheres na transição menopausal e após-menopausa. 2010.120 
f. Trabalho de conclusão de curso (Mestrado) - Universidade de São Paulo, São Paulo, 2010.

PIRES, K. Relação de perdas urinárias e obesidade em mulheres: Avaliação da força muscular do assoalho pélvico.Trabalho de Conclusão de Curso, 83 f. Vitória, 2013.

SARTORI, D., DE SOUZA, J. P., CARNEIRO, P. A influência da obesidade na musculatura do assoalho pélvico em mulheres continentes. Ensaios e Ciência: Ciências Biológicas, Agrárias e da Saúde, São Paulo, v. 15, n. 3, p. 9-23, 2011.

SILVA J. de C., et. al.Grau de força do assoalho pélvico em mulheres incontinentes obesas e não obesas. Revista Ciência \& Saúde, Porto Alegre, v. 4, n. 2, p. 37-44, 2011.

TAMANINI, José T. N., et. al. Analysis of the prevalence of end factors associated with urinary incontinence among elderly people in the municipality of São Paulo, Brazil: SABE Study. (Health, Wellbeing and Aging). Caderno de Saúde Pública, Rio de Janeiro, n.8, p. 1756-1762, 2009

VIECELLI, C., et. al. Obesidade como fator de risco para a falha da cirurgia de Burch. Revista Brasileira de Ginecologia e Obstetrícia., Rio de Janeiro, v. 31, n. 4, p. 182-188, 2009.

WING, R., et. al. Effect of Weight Loss on Urinary Incontinence in Overweight and Obese Women: Results at 12 and 18 Months. J. Urology, v. 183, n.3, p. 1005-1010, 2014.

Enviado: Maio, 2020.

Aprovado: Outubro, 2020. 